\title{
BMJ Open Association between the number of board-certified cardiologists and the risk of in-hospital mortality: a nationwide study involving the Japanese registry of all cardiac and vascular diseases
}

Kihei Yoneyama (D) , ${ }^{1}$ Koshiro Kanaoka, ${ }^{2}$ Satoshi Okayama, ${ }^{2}$ Kunihiro Nishimura, ${ }^{3}$ Michikazu Nakai, ${ }^{4}$ Kunihiro Matsushita, ${ }^{5}$ Yoshihiro Miyamoto, ${ }^{4}$ Keisuke Kida, ${ }^{6}$ Yuki Ishibashi, ${ }^{1}$ Masaki Izumo, ${ }^{1}$ Makoto Watanabe, ${ }^{2}$ Tsunenari Soeda, ${ }^{2}$ Hiroyuki Okura, ${ }^{2}$ Tomoo Harada, ${ }^{1}$ Satoshi Yasuda, ${ }^{7}$ Toyoaki Murohara, ${ }^{8}$ Hisao Ogawa, ${ }^{7}$ Yoshihiko Saito, ${ }^{2}$ Yoshihiro J Akashi (D) ${ }^{1}$

To cite: Yoneyama $\mathrm{K}$, Kanaoka K, Okayama S, et al. Association between the number of board-certified cardiologists and the risk of inhospital mortality: a nationwide study involving the Japanese registry of all cardiac and vascular diseases. BMJ Open 2019;9:e024657. doi:10.1136/ bmjopen-2018-024657

- Prepublication history and additional material for this paper are available online. To view these files, please visit the journal online (http://dx.doi. org/10.1136bmjopen-2018024657).

Received 16 June 2018 Revised 06 June 2019 Accepted 01 August 2019
Check for updates

(C) Author(s) (or their employer(s)) 2019. Re-use permitted under CC BY-NC. No commercial re-use. See rights and permissions. Published by BMJ.

For numbered affiliations see end of article.

Correspondence to Dr Yoshihiro J Akashi; yoakashi-circ@umin.ac.jp

\section{ABSTRACT}

Objectives Although there are 14097 board-certified cardiologists in Japan, it is unknown whether the number of institutional board-certified cardiologists is related to the prognosis of cardiovascular disease patients.

Design Cross-sectional analysis.

Setting Data were collected from the nationwide database of acute care hospitals in Japan (2371 hospitals) between 2012 and 2013.

Participants A total of 1422703 consecutive patients were initially included in this study, but 518610 patients were excluded due to age $<18$ years, missing data or prior hospitalisations; therefore, 896171 patients comprised the final sample population.

Main outcome measures The primary outcome was in-hospital mortality due to any cause. For the per-hospital analysis, Poisson regression models were used to estimate the association of board-certified cardiologists with inhospital mortality, adjusted for hospital facilitation. For the per-patient analysis, hierarchical logistic regression models were used to estimate the ORs of the number of institutional board-certified cardiologists, adjusted for patient demographics, diagnoses, therapies and hospital facilities.

Results The regression model of the per-hospital analysis indicated that the number of board-certified cardiologists was associated with a lower rate ratio of in-hospital mortality (rate ratio, $0.988 ; 95 \% \mathrm{Cl} 0.983$ to 0.993; $p<0.01$ ). The per-patient analysis indicated that the median age was 73 years and the in-hospital mortality rate was $11.7 \%$. The regression model indicated that the presence of more board-certified cardiologists was associated with a lower risk of in-hospital mortality (OR, $0.980 ; 95 \% \mathrm{Cl} 0.975$ to $0.986 ; \mathrm{p}<0.01$ ) after adjustments for hospital facilities, patient characteristics and treatments.

Conclusions Among cardiovascular disease patients admitted to acute care hospitals in Japan, the presence of more board-certified cardiologists was associated with a lower risk of in-hospital mortality. These results
Strengths and limitations of this study

We performed the largest nationwide study examining the association between in-hospital mortality and number of board-certified cardiologists in Japan.

- Using data from nationwide databases, we found that having more board-certified cardiologists at a hospital was associated with a lower risk of inhospital mortality, independent of the facility and patient characteristics.

- Acute care hospitals with cardiology beds were only included in the present study; therefore, the results may not reflect other institutions.

have implications for national and institutional strategies for determining the required number of board-certified cardiologists.

\section{INTRODUCTION}

Cardiovascular diseases are among the leading global and regional causes of death worldwide. Deaths from cardiovascular disease have increased globally by $41 \%$ between 1990 and 2013, accounting for an estimated 17.3 million of the 54 million total deaths in 2013. ${ }^{12}$ However, research of cardiovascular diseases by physicians, researchers and regional heart associations has resulted in an abundance of evidence-based medical therapies such as aspirin, beta-blockers, coronary and valve interventions, device therapies and emerging imaging techniques. ${ }^{3-8}$ The expanded use of these therapies is increasing the quality of cardiac medical care and, hopefully, decreasing mortality rates. Physicians with more experience are generally 
A

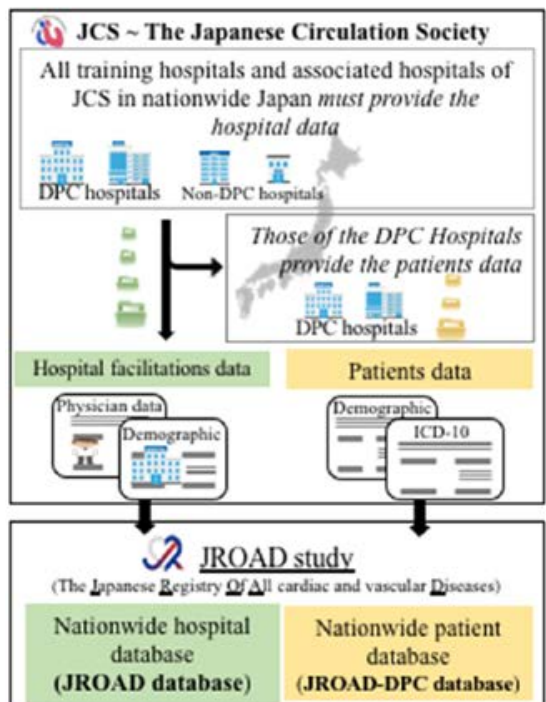

B

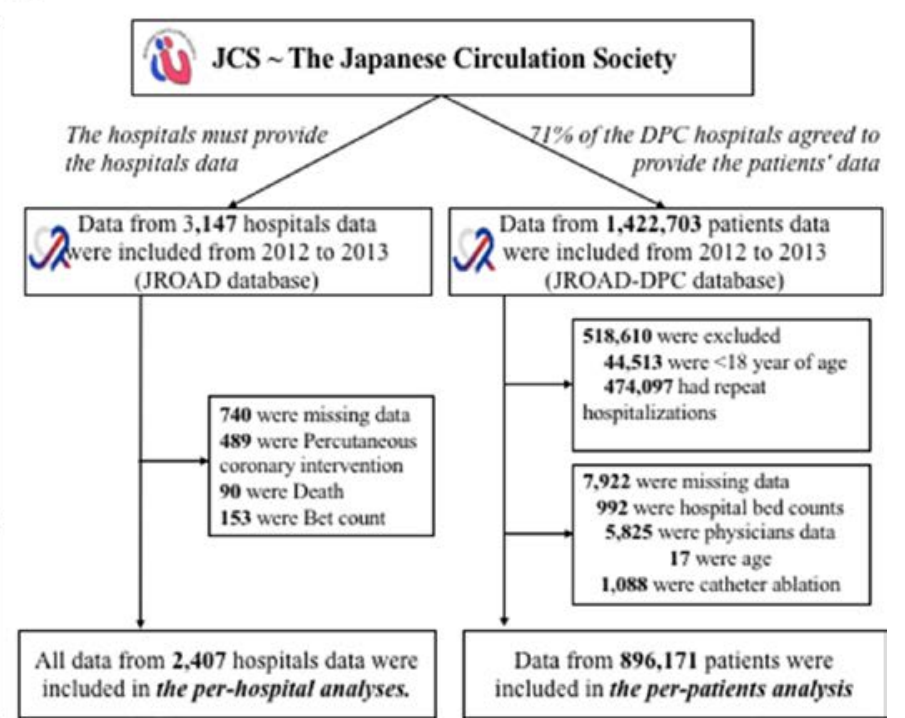

Figure 1 Flowchart of the present study. (A) The JROAD study consisted of a large dataset obtained through the Japanese administrative case-mix DPC system in which individual patient records are continuously collected systematically nationwide using a uniform reporting format. Physicians are required to provide complete diagnostic code data until the time of patient discharge. Nearly all DPC hospitals with cardiovascular beds meet the Japanese Circulation Society (JCS) requirements. All training and associated hospitals of the JCS must provide institutional data (ie, hospital bed count, number of board-certified cardiologists and so on). DPC hospitals also provide patient data (ie, age, sex, ICD-10 codes and so on). (B) A flowchart describing the two datasets used for the per-hospital and per-patient analyses to evaluate the association between boardcertified cardiologists and in-hospital mortality. DPC, diagnostic procedure combination; ICD-10, International Classification of Diseases and Injuries, 10th revision; JROAD, Japanese registry of all cardiac and vascular diseases.

considered to have accumulated knowledge and skills during practice; therefore, they can provide high-quality patient care.

Board-certified cardiologists are required to complete intense training programmes and successfully complete examinations. Additionally, the certification is usually effective for only a limited time (eg, 5 years in Japan); therefore, cardiologists need to remain current with the latest clinical advances and successfully complete recertification requirements. Although there are 14097 boardcertified cardiologists in Japan, there is little information regarding whether the number of institutional boardcertified cardiologists is related to better prognoses for patients with cardiovascular diseases in clinical practice. Addressing this question with a randomised, controlled trial (eg, a clustered randomised trial contrasting the care of patients with cardiovascular disease by board-certified cardiologists compared with physicians not certified in cardiology) is not feasible and might be unethical. ${ }^{9}$ Therefore, we conducted an observational study using a large, nationwide dataset, the Japanese registry of all cardiac and vascular diseases (JROAD), to determine whether the number of institutional board-certified cardiologists is related to better prognoses for patients with cardiovascular diseases. The JROAD is a nationwide database that includes hospital and patient characteristics, principal diagnoses, comorbidities at admission, complications after admission, procedures during hospitalisation and discharge status.

\section{MATERIALS AND METHODS}

\section{Patients and public involvement}

The JROAD database is a nationwide prospective registry that was designed to assess the clinical activity of each Japanese institution providing cardiovascular care and to provide adequate feedback to teaching hospitals to improve patient care. A detailed description of the database design and methods has been previously published. ${ }^{10}$ Briefly, the Japanese Circulation Society (JCS) developed the JROAD database, which includes each hospital's demographics since 2004, and the JROAD diagnosis procedure combination (JROAD-DPC) nationwide database, which includes data from the Japanese DPC/Per Diem Payment System (DPC/PDPS) since 2014. The DPC database is a mixed-case classification system linked with a lump-sum payment system that was launched in 2002 by the Ministry of Health, Labour, and Welfare of Japan. ${ }^{11}$ Compared with other registry databases, the Japanese DPC database enables researchers to conduct nationwide studies of descriptive and/or analytical epidemiology in real-world clinical settings. The DPC database includes data regarding the following elements: demographics for each patient (eg, age and sex); principal diagnoses (coded according to the International Classification of Diseases and Injuries, 10th revision (ICD-10)); comorbidities at admission (ICD-10 code); complications after admission (ICD-10 code); procedures, including surgery, medications and devices used during hospitalisation; length of stay; discharge status; and medical expenses. ${ }^{10-13}$ 


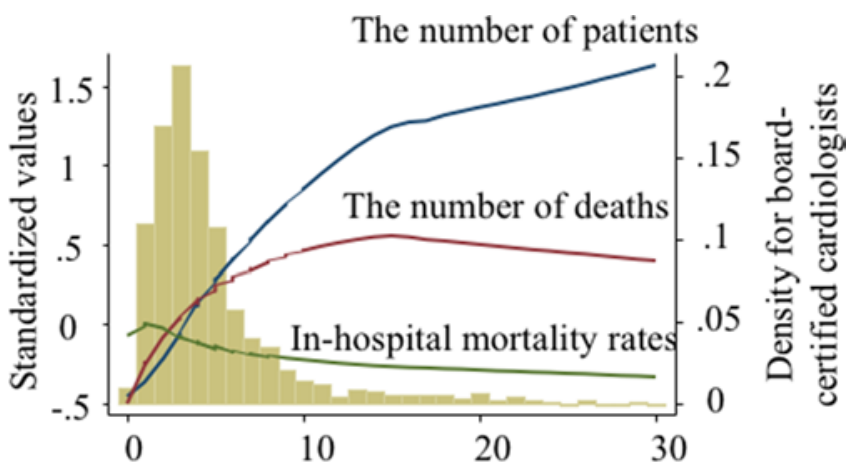

The number of board-certified cardiologists

Figure 2 The association between board-certified cardiologists and in-hospital mortality rates for cardiovascular diseases according to the per-hospital analyses. A plot of locally weighted smoothing curves (LOWESS) using unadjusted standardised values of the numbers of patients (blue line), deaths (red line) and in-hospital mortality rates (green line), including a histogram of the distributions of board-certified cardiologists (yellow bars). Unadjusted standardised values were calculated by dividing the differences between the observed values and the sample means by the corresponding SD. Hospitals with more boardcertified cardiologists also had more patients, more deaths and lower in-hospital mortality rates than those with fewer board-certified cardiologists. In-hospital morality rates were calculated by dividing the total number of deaths by the total number of patients.

Institutions using the DPC system encompass a wide variety of centres, including academic, urban and rural hospitals. ${ }^{10}{ }^{14}$ All data included in this study were from hospitalised patients with clinically apparent cardiovascular diseases. This research was approved by the institutional review boards at all participating institutions. Each hospital anonymised the patients' identities using code change equations for the original JROAD-DPC data. Individual data (including age, sex and bed count) were sent to the Ministry of Health, Labour, and Welfare, which notified the patients through websites or posters at each hospital that their information was being collected for this study.

\section{Study design and sampling}

We conducted a cross-sectional study using both the JROAD and JROAD-DPC data obtained between 1 April 2012 and 31 March 2013. A total of 2407 hospitals were included in the JROAD database; of those, 1247 (52\%) hospitals adapted the DPC system and agreed to participate in the JROAD-DPC database (figure 1). Characteristics of JROAD-DPC data from participating and non-participating hospitals and ICD-10 diagnosis codes were previously summarised ${ }^{10}$ and are presented in online supplementary eTable1. A total of 1422703 consecutive patients were initially included in this study; however, 518610 patients were excluded due to missing data, age younger than 18 years, prior hospitalisations, or their choice to opt-out of the study. Patients with no missing variables were included in the analyses $(n=896171)$.
Board-certified cardiologists were defined as boardcertified members of the JCS (http://www.j-circ.or.jp/ english/about/outline.html). Board-certified cardiologists must be regular members of the JCS for a minimum of 6 years, must be board-certified members of the Japanese Society of Internal Medicine, Japan Surgical Society, Japan Pediatric Society, Japanese Association of Thoracic Surgery or the American Society of Internal Medicine, must have completed 3 years of training at a certified institution and must have successfully completed the examination offered by the JCS. Each hospital annually confirmed the certifications of their board-certified cardiologists, including physician names, but they only provided the total number of board-certified cardiologists per hospital to the JROAD database. Therefore, we were unable to create a merged dataset to identify which physician was responsible for treating which patients. The number of non-board-certified cardiologists per year was defined as the total number of cardiologists within a given hospital minus the number of board-certified cardiologists within the hospital. The ratio of beds to boardcertified cardiologists was calculated as the bed count per hospital divided by the number of board-certified cardiologists of that same hospital. A higher index represents a larger number of beds per board-certified cardiologist.

\section{Outcomes}

The primary outcome was the occurrence of in-hospital mortality from any cause. The JROAD-DPC data provided a relatively complete perspective regarding mortality during hospitalisation.

\section{Statistical analysis}

The numbers of board-certified cardiologists and the ratios of beds to board-certified cardiologist were divided into quartiles. The per-hospital analysis determined the association between the number of board-certified cardiologists and the in-hospital mortality risk of patients hospitalised for cardiovascular diseases in the cardiology department. Multilevel mixed-effects Poisson regression models were used to consider the random effects of hospital variation in the JROAD database. The rate ratio of the board-certified cardiologists was adjusted for hospital demographics, including the number of nonboard-certified cardiologists, hospital beds, presence of cardiac surgery services, paediatrics and eight regional divisions (eg, the Kanto district), and for the number of cardiac therapies (percutaneous coronary intervention, coronary artery bypass grafting, congenital surgery, catheter ablation, implantable cardioverter defibrillator and cardiac resynchronisation) per year.

For the per-patient analysis, we excluded all patients who were younger than 18 years and those who had prior hospitalisations according to the DPC reports. If patients were hospitalised more than once during the study period, then we used only the data associated with the last admission; we also counted the number of prior hospitalisations. The characteristics of patients who died 
in the hospital were compared with the characteristics of the patients who survived using both a non-parametric test for continuous variables and a $\chi^{2}$ test for categorical variables.

To assess the association of board-certified cardiologists with in-hospital mortality, we used hierarchical logistic regression models to estimate the ORs for in-hospital mortality. Each of the hierarchical logistic regression model variables included a hierarchy level (hospital) that considered the random effects of hospital variations. All estimates were adjusted for hospital demographics, patient demographics (age, sex, Charlson comorbidity index, and the presence of angina pectoris, myocardial infarction, atrial fibrillation, heart failure, aorta disease, cardiac arrest, pulmonary thromboembolism, primary pulmonary hypertension, tetralogy of Fallot and incidental non-cardiac diseases such as stroke, pneumonia, acute renal failure and gastrointestinal bleeding during and after hospitalisation), number of prior hospitalisations and number of cardiac therapies per year. The adjusted probabilities of in-hospital mortality were calculated using the 'marginsplot' for multiple hierarchical logistic regression models using STATA. All analyses were performed using STATA statistical software V.14.

Table 1 Association of board-certified cardiologists and in-hospital mortality rates according to the per-hospital analysis

\begin{tabular}{|c|c|c|}
\hline \multirow[b]{2}{*}{ Hospital $(n=2371)$} & \multicolumn{2}{|l|}{ Poisson regression } \\
\hline & $\begin{array}{l}\text { Adjusted rate ratio } \\
(95 \% \mathrm{Cl}) \dagger\end{array}$ & $\begin{array}{l}\text { Coefficient } \\
\text { (SE) }\end{array}$ \\
\hline \multicolumn{3}{|c|}{ Board-certified cardiologists } \\
\hline Continuous value & $0.971(0.969 \text { to } 0.973)^{\star}$ & $-0.030(0.001)^{\star}$ \\
\hline \multicolumn{3}{|l|}{ Categorical value } \\
\hline Q1 (<2) & Reference & \\
\hline Q2 (3) & $0.773(0.756 \text { to } 0.790)^{*}$ & $-0.258(0.011)^{*}$ \\
\hline Q3 (4) & $0.800(0.7820 \text { to } 0.819)^{\star}$ & $-0.223(0.012)^{\star}$ \\
\hline Q4 (5-6) & $0.732(0.715 \text { to } 0.749)^{*}$ & $-0.312(0.012)^{*}$ \\
\hline Q5 (>7) & $0.562(0.548 \text { to } 0.575)^{\star}$ & $-0.577(0.012)^{\star}$ \\
\hline \multicolumn{3}{|c|}{ Ratio of hospital beds to board-certified cardiologists } \\
\hline Continuous value $\neq$ & $1.020(1.019 \text { to } 1.021)^{\star}$ & $0.020(0.001)^{*}$ \\
\hline \multicolumn{3}{|l|}{ Categorical value } \\
\hline Q1 $(<40)$ & Reference & \\
\hline Q2 (40-58) & $1.389(1.359 \text { to } 1.420)^{*}$ & $0.328(0.011)^{\star}$ \\
\hline Q3 (59-77) & $1.407(1.376 \text { to } 1.439)^{\star}$ & $0.341(0.011)^{\star}$ \\
\hline Q4 (78-104) & $1.563(1.529 \text { to } 1.599)^{*}$ & $0.447(0.011)^{\star}$ \\
\hline Q5 (>105) & $1.800(1.760 \text { to } 1.841)^{\star}$ & $0.588(0.011)^{\star}$ \\
\hline
\end{tabular}

${ }^{*} \mathrm{P}<0.01$.

†The rate ratio was adjusted for the number of non-boardcertified cardiologists, hospital beds, the presence of cardiac surgery and paediatric services, and eight regional divisions in Japan.

‡ORs of 10 hospital beds to board-certified cardiologists.

$\mathrm{Q}$, quartile.
RESULTS

\section{Hospital characteristics for the per-hospital analysis}

Data from a total of 2407 hospitals were included in the per-hospital analysis. There were 11687 board-certified cardiologists at acute hospitals in Japan between 2012 and 2013. Hospital characteristics are shown in online supplementary eTable2. The overall median bed count was 350 , the number of institutional board-certified cardiologists was 4 , the ratio of beds to board-certified cardiologists was 68 and median in-hospital mortality was $0.03 \%$ per hospital. Additionally, $80 \%$ of the hospitals had coronary care units and $56 \%$ had cardiac surgery services. Moreover, online supplementary eTable3 shows the hospital characteristics. Hospitals that provided data to the JROAD-DPC more often had larger bed counts, had more board-certificate cardiologists, performed invasive coronary angiography and performed device therapy than hospitals that did not provide data to the JROAD-DPC.

\section{Association of board-certified cardiologists with in-hospital mortality according to the per-hospital analysis}

Hospitals with a larger number of board-certified cardiologists were likely to have a larger number of patients and a larger number of deaths than those with fewer
A

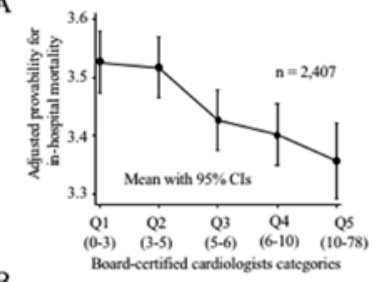

B

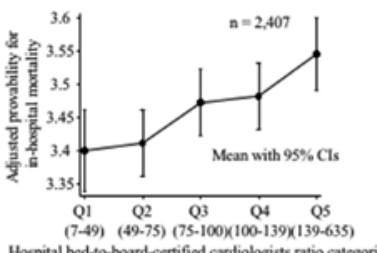

C

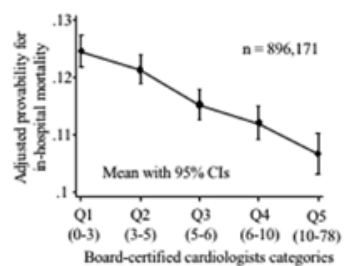

D

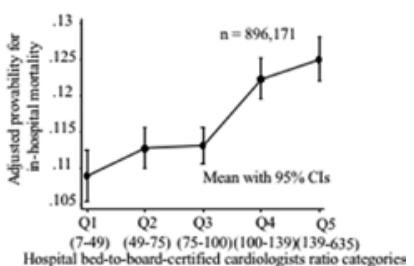

Figure 3 In-hospital mortality rates in relation to boardcertified cardiologists for cardiovascular disease. (A) The linear association of the mean adjusted probability for in-hospital mortality across the quartiles of the number of board-certified cardiologists. (B) The linear association of the mean adjusted probability for in-hospital mortality across the quartiles of the ratios of beds to board-certified cardiologists. Adjusted probabilities were calculated using the 'margins' command and the multilevel mixed-effects Poisson regression analyses (table 1 ). (C) The linear association of the mean adjusted probability for in-hospital mortality across the quartiles of the number of board-certified cardiologists. (D) The linear association of the mean adjusted probability for in-hospital mortality across the quartiles of the ratios of beds to board-certified cardiologists. The bars indicate 95\% Cls. The adjusted probability was calculated using the margins command in STATA and was adjusted as indicated in table 3. $(A, B)$ represent the per-hospital analysis, and $(C, D)$ represent the per-patient analysis. 
Table 2 Characteristics of the patients according to in-hospital death and patient survival

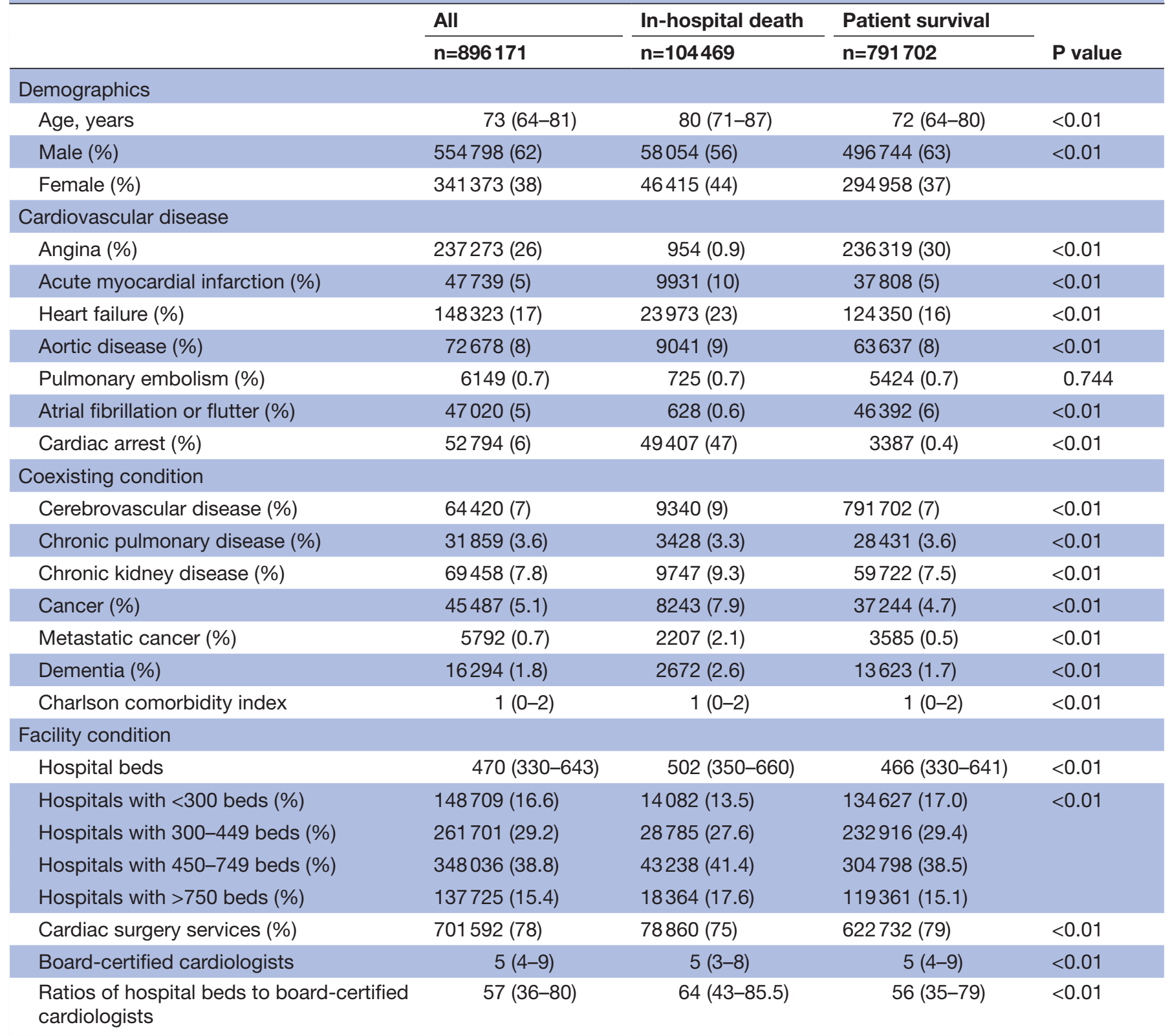

Values are either medians with IQRs (Q1 to Q3) or percentages (when indicated). Baseline characteristics were compared using the Wilcoxon rank-sum test for continuous variables and the $\chi^{2}$ test for categorical variables.

board-certified cardiologists; however, they were also likely to have lower mortality rates (figure 2). The multilevel mixed-effects Poisson regression model indicated that the number of institutional board-certified cardiologists was associated with a lower rate ratio of in-hospital mortality (rate ratio, 0.988; (95\% CI 0.983 to $0.993)$ per 1 increment of the number of institutional board-certified cardiologists; $\mathrm{p}<0.01$ ) after adjustments for the number of non-board-certified cardiologists, hospital beds, presence of cardiac surgery and paediatric services, eight regional divisions and number of therapies (table 1 and figure 3A,B). Similarly, the ratio of beds to board-certified cardiologists was associated with an increased risk of in-hospital mortality (rate ratio, 1.006 (95\% CI 1.003 to 1.009$)$ per 1 increment of the 10 beds to board-certified cardiologists ratio; $\mathrm{p}<0.01)$ after the adjustments.

\section{Patient characteristics used for the per-patient analysis}

The final study sample included 896171 patients with cardiovascular diseases who were admitted to DPC hospitals. The clinical presentation of the patients and the facility characteristics are shown in table 2. Overall, the median age was 73 years, and the male:female ratio was 62:38. The most common cardiovascular diseases of patients admitted to hospitals were angina (26\%), heart failure (17\%), aortic disease $(8 \%)$ and myocardial infarction $(5 \%)$. Patients who died in the hospital were 
Table 3 Association between the number of board-certified cardiologists and in-hospital mortality rates according to the perpatient analysis

\begin{tabular}{|c|c|c|c|}
\hline In-hospital mortality & \multicolumn{3}{|c|}{ Multilevel mixed-effects logistic regression and adjusted OR $(95 \% \mathrm{Cl})$} \\
\hline (No of events: 104 469/896 171) & Adjusted for facility & $\begin{array}{l}\text { Adjusted for clinical } \\
\text { characteristics§ }\end{array}$ & Adjusted for treatments $\emptyset$ \\
\hline \multicolumn{4}{|l|}{ Board-certified cardiologists } \\
\hline Continuous value & $0.976(0.97 \text { to } 0.982)^{\star}$ & $0.979(0.973 \text { to } 0.985)^{\star}$ & $0.98(0.975 \text { to } 0.986)^{\star}$ \\
\hline \multicolumn{4}{|l|}{ Categorical value } \\
\hline Q1 (0-3; n=179234) & Reference & Reference & Reference \\
\hline Q2 (3-5; n=179234) & $0.981(0.948 \text { to } 1.015)^{*}$ & $0.949(0.912 \text { to } 0.988)^{*}$ & $0.948(0.911 \text { to } 0.987)^{*}$ \\
\hline Q3 (5-6; n=179234) & $0.902(0.856 \text { to } 0.950)^{\star}$ & $0.841(0.793 \text { to } 0.891)^{\star}$ & $0.850(0.802 \text { to } 0.900)^{*}$ \\
\hline Q4 (6-10; $n=179234)$ & $0.848(0.792 \text { to } 0.907)^{*}$ & $0.783(0.729 \text { to } 0.841)^{*}$ & $0.799(0.744 \text { to } 0.858)^{*}$ \\
\hline Q5 (10-78; n=179235) & $0.834(0.764 \text { to } 0.910)^{\star}$ & $0.715(0.652 \text { to } 0.784)^{\star}$ & $0.720(0.656 \text { to } 0.790)^{*}$ \\
\hline \multicolumn{4}{|c|}{ Ratio of hospital beds to board-certified cardiologists } \\
\hline Continuous value ${ }^{\star \star}$ & $1.012(1.008 \text { to } 1.015)^{\star}$ & $1.012(1.008 \text { to } 1.015)^{\star}$ & $1.012(1.008 \text { to } 1.015)^{\star}$ \\
\hline \multicolumn{4}{|l|}{ Categorical value } \\
\hline Q1 (7-49; n=185371) & Reference & Reference & Reference \\
\hline Q2 (49.75; n=179944) & 0.987 (0.920 to 1.059 ) & $1.084(1.004$ to 1.17$) \dagger$ & 1.078 (0.999 to 1.163$)$ \\
\hline Q3 (75-100; n=180 097) & $1.096(1.012$ to 1.187$) \dagger$ & 1.095 (1.010 to 1.187$) \dagger$ & $1.084(1.00$ to 1.176$) \dagger$ \\
\hline Q4 (100-139; n=171807) & $1.264(1.160 \text { to } 1.376)^{*}$ & $1.278(1.177 \text { to } 1.388)^{*}$ & $1.279(1.178 \text { to } 1.389)^{*}$ \\
\hline Q5 (139-635; n=178952) & $1.306(1.191 \text { to } 1.433)^{\star}$ & $1.352(1.242 \text { to } 1.472)^{\star}$ & $1.339(1.23 \text { to } 1.458)^{*}$ \\
\hline
\end{tabular}

${ }^{*} \mathrm{P}<0.01$.

$+\mathrm{P}<0.05$

$\ddagger$ Model included the number of non-board-certified cardiologists, hospital beds, coronary care units, cardiac surgery services and eight regional divisions.

§Model included age, sex, Charlson comorbidity index, and rates of angina pectoris, myocardial infarction, atrial fibrillation, heart failure, aorta disease, cardiac arrest, pulmonary thromboembolism, primary pulmonary hypertension, and tetralogy of Fallot, and rates of non-cardiac disease (stroke, pneumonia, acute renal failure and gastrointestinal bleeding) after hospitalisations and the number of prior hospitalisations, in addition to the facility model.

ๆModel included percutaneous coronary interventions, coronary artery bypass grafts, catheter ablations, implantable cardioverterdefibrillators and cardiac resynchronisation therapy in addition to the clinical characteristics model.

${ }^{* *} \mathrm{OR}$ of ratios of 10 hospital beds to board-certified cardiologists.

Q, quartile.

older and exhibited a higher prevalence of myocardial infarction, heart failure and cardiac arrest (all $\mathrm{p}<0.01$ ). Additionally, higher in-hospital mortality was associated with hospitals with higher mean bed counts (502 $(350-660)$ vs $466(330-641) ; \mathrm{p}<0.01)$ and with hospitals with fewer board-certified cardiologists (5 (3-8) vs $5(4-9) ; \mathrm{p}<0.01)$. Higher in-hospital mortality rates were also associated with hospitals with larger ratios of beds to board-certified cardiologists (64 (43-85.5) vs 56 $(35-79) ; \mathrm{p}<0.01)$.

\section{Association of board-certified cardiologists with in-hospital mortality according to the per-patient analysis}

The per-patient analysis indicated a total in-hospital mortality rate of $11.7 \%$. The multilevel mixed-effects logistic regression model showed that the number of institutional board-certified cardiologists was related to lower in-hospital mortality (OR, 0.980; 95\% CI 0.975 to $0.986 ; \mathrm{p}<0.01)$ after adjusting for facilities, patient characteristics and treatments (table 3, online supplementary eTable 4 and figure $3 \mathrm{C}, \mathrm{D})$. The ratio of beds to board-certified cardiologists was associated with a higher risk for in-hospital mortality (OR, 1.012 (95\% CI 1.008 to 1.015 ) per 1 increment of ratios of 10 beds to board-certified cardiologists; $\mathrm{p}<0.01$ ) after adjusting for facilities, patient characteristics and treatments.

An analysis of the number of institutional board-certified cardiologists and in-hospital mortality according to variables is shown in figure 4. Hierarchical logistic regression models demonstrated significant interactions between the number of institutional board-certified cardiologists and patient age $(p<0.01)$, number of hospital beds $(p<0.01)$, angina $(\mathrm{p}<0.01)$, myocardial infarction $(\mathrm{p}=0.012)$, aortic diseases $(p=0.01)$ and cardiac arrest $(p<0.01)$. The association of the number of institutional board-certified cardiologists with the lower risk of in-hospital mortality was more evident for patients admitted to hospitals with fewer than 300 beds than for those hospitalised at larger institutions (hospitals with $>750$ beds and hospitals with $450-749$ beds vs hospitals with $<300$ beds: $\mathrm{p} \leq 0.01$ ). 
Number of board-certified cardiologists, $\mathrm{n}$

No. of event/No. at risk Adjusted odds ratio $(95 \% \mathrm{Cl})$

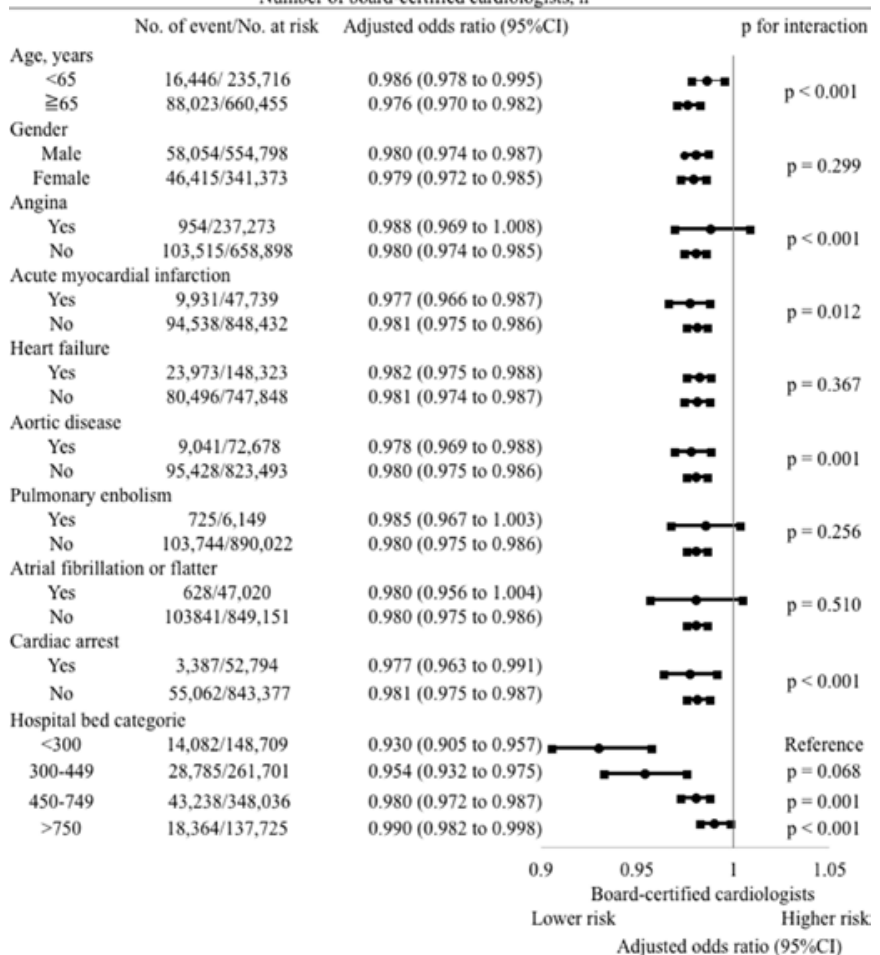

Figure 4 Adjusted ORs and 95\% Cls for in-hospital mortality in relation to board-certified cardiologists for cardiovascular disease The interaction of hierarchical logistic regression test results indicated that there the number of board-certified cardiologists had a significant impact on patient characteristics and hospital volume. An OR $<1.0$ represents a decrease in the risk of in-hospital mortality according to the number of board-certified cardiologists. The OR is indicated by the dot, and the lines represent the $95 \%$ $\mathrm{Cls}$. ORs were adjusted for facilities, patient characteristics and treatments, as indicated in table 3 .

\section{DISCUSSION}

\section{Principal findings}

Using data from two large nationwide databases, we found that a large number of board-certified cardiologists at a hospital were associated with a lower risk of in-hospital mortality, independent of facilities and patient characteristics. The lower risk of mortality was consistently observed for each cardiovascular disease and more evident for smaller hospitals. The balance between the bed count and boardcertified cardiologists (eg, a high-volume hospital with the relative shortage of board-certified cardiologists) should be considered to improve the care of cardiovascular disease patients (figure 5).

\section{Study strengths and limitations}

Data of 2371 hospitals and 896171 patients from the JROAD and JROAD-DPC databases were analysed during this study, thus making it one of the largest cross-sectional and retrospective studies of nationwide cardiac health outcomes. One limitation of our study was that we only included DPC hospitals with cardiovascular beds that met the JCS requirements. Another limitation was the small number of patients with specific diseases. Although the variable analyses may
Board-certified cardiologists

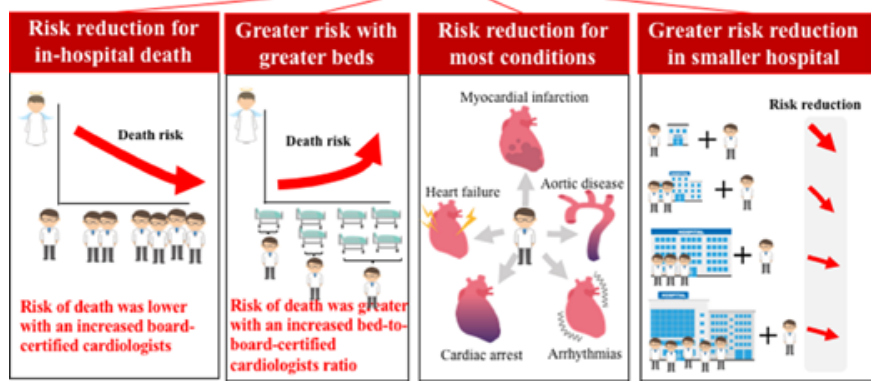

Figure 5 The main findings from this study. A larger number of board-certified cardiologists at a hospital was associated with a lower risk of in-hospital mortality. A higher ratio of beds to board-certified cardiologists at a hospital was associated with a greater risk of death. Board-certified cardiologists were more likely to provide benefits to patients by preventing deaths due to cardiovascular diseases. The risk of in-hospital mortality was attenuated by increasing the hospital volume. These observations suggest that board-certified cardiologists may improve patient outcomes, and that the balance between bed numbers and board-certified cardiologists should be considered to improve patient care.

have lacked the statistical power to demonstrate clinically meaningful differences in mortality among patients with angina and atrial fibrillation (lower mortality rates) and with pulmonary embolism (fewer cases), our findings indicated that an overall increase in board-certified cardiologists may prevent in-hospital mortality for patients with cardiovascular diseases.

\section{Comparison of our results and those of other studies}

Previous research has shown that more experience accounts for better outcomes, as reported by studies examining acute myocardial infarction, ${ }^{15-17}$ percutaneous coronary intervention, ${ }^{18}$ implantable cardioverter-defibrillators ${ }^{19} 20$ and heart failure. ${ }^{21-23}$ Unlike most previous studies, our study confirmed that a lower risk of mortality and a larger number of board-certified cardiologists was consistently seen for most cardiovascular diseases. Data from the ICD registry indicated that in the USA, implantable cardioverter-defibrillator implantations performed by a non-electrophysiologist were associated with a higher risk of procedural complications and a lower likelihood of receiving cardiac resynchronisation therapy when compared with patients whose implantable cardioverter-defibrillators were implanted by an electrophysiologist. ${ }^{19}$ Our findings are consistent with these observations. Board-certified cardiologists may be more likely to utilise guidelines, clinical teams, disease management, discharge programmes and patient education seminars when implementing treatment, all of which improve patient outcomes. ${ }^{1524-28}$

\section{Meaning of the study: possible explanations of the results}

Although our observational study did not determine causality, there were a few plausible mechanisms underlying the observed lower mortality risk related to the number of board-certified cardiologists. For example, the shortage of 
board-certified cardiologists could delay optimal therapies or prevent eligible patients from receiving potentially lifesaving therapies. Additionally, our study found that despite the presence of board-certified cardiologists lowering the risk of patient mortality, we observed heterogeneity among hospitals with differing bed counts, with smaller hospitals having lower mortality rates. This may suggest that patients in smaller hospitals are generally less severely ill than those in larger hospitals; therefore, smaller-volume hospitals may have better patient outcomes. Reports have suggested a recent acceleration in the development of specialty hospitals with a focus on narrow procedural areas, and proponents see these specialty hospitals as an opportunity to improve patient care. ${ }^{29-31}$ In contrast, data from a study of Medicare administrative claims in the USA have been inconsistent for high-volume hospitals and lower mortality rates, which could be due to the mismanagement of medicinal conditions by multiple healthcare professionals, including physicians, nurses and other clinical staff. ${ }^{32}$ However, that study also found that the relationship was attenuated for larger hospital volumes, and that the highest-volume hospitals were no longer significantly associated with higher mortality rates. This could suggest that the highest-volume hospitals had more experienced cardiologists. According to our study, hospitals with more beds exhibited higher rates of mortality, as did hospitals with higher ratios of beds to board-certified cardiologists.

\section{Meaning of the study: implications for clinical care}

Our findings have clear implications. First, our findings indicate evidence that board-certified cardiologists are beneficial to clinical cardiac care by reducing the risk of patient death. Therefore, we suggest that current board-certified cardiologists should successfully maintain competence, develop new skills and gain knowledge in their current field. Second, policymakers should focus on integrating the strategies that have been identified to improve patient care in hospitals with a shortage of board-certified cardiologists. Third, because all-cause mortality can be used to benchmark quality and compare hospital performance, our findings regarding the balance between bed counts and board-certified cardiologists should be considered to improve patient care.

\section{Unanswered questions and future research}

Additional findings of lower ORs for elderly patients (65 years or older) and for patients with myocardial infarction, aortic disease and cardiac arrest compared with patients without these conditions could provide further information regarding these specific patient populations.

\section{CONCLUSIONS}

According to nationwide hospital databases, a larger number of institutional board-certified cardiologists was associated with a lower risk of in-hospital mortality, independent of potential patient confounders. This relationship was particularly evident at smaller hospitals. Our results have implications for national and institutional strategies because they can help determine the required number of board-certified cardiologists per hospital to reduce mortality rates.

\section{Author affiliations}

${ }^{1}$ Division of Cardiology, Department of Internal Medicine, St. Marianna University School of Medicine, Kawasaki, Japan

${ }^{2}$ Department of Cardiovascular Medicine, Nara Medical University, Kashihara, Nara, Japan

${ }^{3}$ Preventive Medicine and Epidemiology Informatics, Center for Cerebral and Cardiovascular Disease Information, National Cerebral and Cardiovascular Center,

Suita, Japan

${ }^{4}$ Department of Statistics and Data Analysis, Center for Cerebral and Cardiovascular Disease Information, National Cerebral and Cardiovascular Center, Suita, Osaka, Japan

${ }^{5}$ Department of Epidemiology, Johns Hopkins Bloomberg School of Public Health, Baltimore, Maryland, USA

${ }^{6}$ Department of Pharmacology, St. Marianna University School of Medicine, Kawasaki, Kanagawa, Japan

${ }^{7}$ Department of Cardiovascular Medicine, National Cerebral and Cardiovascular Center, Suita, Osaka, Japan

${ }^{8}$ Department of Cardiology, Nagoya University Graduate School of Medicine Faculty of Medicine, Nagoya, Japan

Acknowledgements The authors thank Yoko Sumita and the other investigators, staff, and participants in the JROAD study for their valuable contributions. A full list of institutions can be found at http://jroadinfo.ncvc.go.jp.

Contributors The corresponding author attests that all listed authors meet the authorship criteria and that no others meeting the criteria have been omitted. $\mathrm{KY}$, KKa, SO, KM, YM, KN, YI, MI, MW, TS, HOK, TH, TM, YS and YJA developed the study concept and study design. KY, KKa, S0, KN and MN performed the data analysis and interpretation under the supervision of KM, YM, KKi, Ml, MW, TS, TH, HOg, SY, TM and YS. KY, KM, MI, KKa, SY, YS and YJA drafted the manuscript and all the coauthors provided critical revisions. All the authors approved the final version of the manuscript for submission.

Funding KY has reported receiving personal fees from Sanofi Co. Ltd. SY reported personal fees received from Takeda Pharmaceutical Co. Ltd., Daiichi Sankyo Co. Ltd. and Abbott Co. Ltd. YS reported personal fees received from Mitsubishi Tanabe Pharma Corporation, Otsuka Pharmaceutical Co. Ltd., Daiichi Sankyo Co. Ltd., Novartis Pharma K.K., Pfizer Japan Inc., Nippon Boehringer Ingelheim Co. Ltd., Ono Pharmaceutical Co. Ltd., St. Jude Medical Japan Co., Ltd., Bayer Holding Ltd., Terumo Corporation, Kyowa Hakko Kirin Co. Ltd., Dainippon Sumitomo Pharma Co. Ltd., Astellas Pharma Inc., Takeda Pharmaceutical Co. Ltd., Teijin Pharma Ltd., Shionogi \& Co. Ltd., Kowa Pharmaceutical Co. Ltd., Actelion Pharmaceuticals Japan Ltd. and the endowment department of MSD K.K.

Competing interests None declared.

Patient consent for publication Not required.

Ethics approval The present research was designed by the authors and approved by St. Marianna University of Medicine.

Provenance and peer review Not commissioned; externally peer reviewed.

Data availability statement № data are available.

Open access This is an open access article distributed in accordance with the Creative Commons Attribution Non Commercial (CC BY-NC 4.0) license, which permits others to distribute, remix, adapt, build upon this work non-commercially, and license their derivative works on different terms, provided the original work is properly cited, appropriate credit is given, any changes made indicated, and the use is non-commercial. See: http://creativecommons.org/licenses/by-nc/4.0/.

\section{ORCID iDs}

Kihei Yoneyama http://orcid.org/0000-0002-3952-6398

Yoshihiro J Akashi http://orcid.org/0000-0001-7670-7326 


\section{REFERENCES}

1 Global Burden of Metabolic Risk Factors for Chronic Diseases Collaboration. Cardiovascular disease, chronic kidney disease, and diabetes mortality burden of cardiometabolic risk factors from 1980 to 2010: a comparative risk assessment. Lancet Diabetes Endocrinol 2014;2:634-47.

2 Benjamin EJ, Blaha MJ, Chiuve SE, et al. Heart disease and stroke Statistics-2017 update: a report from the American heart association. Circulation 2017;135:e146-603.

3 Ogawa H, Nakayama M, Morimoto T, et al. Low-dose aspirin for primary prevention of atherosclerotic events in patients with type 2 diabetes: a randomized controlled trial. JAMA 2008;300:2134-41.

4 Packer M, Bristow MR, Cohn JN, et al. The effect of carvedilol on morbidity and mortality in patients with chronic heart failure. U.S. carvedilol heart failure Study Group. N Engl J Med 1996;334:1349-55.

5 Serruys PW, Morice M-C, Kappetein AP, et al. Percutaneous coronary intervention versus coronary-artery bypass grafting for severe coronary artery disease. N Engl J Med 2009;360:961-72.

6 Holmes DR, Brennan JM, Rumsfeld JS, et al. Clinical outcomes at 1 year following transcatheter aortic valve replacement. JAMA 2015;313:1019-28.

7 Young JB, Abraham WT, Smith AL, et al. Combined cardiac resynchronization and implantable cardioversion defibrillation in advanced chronic heart failure: the miracle ICD trial. JAMA 2003;289:2685-94.

8 Min JK, Leipsic J, Pencina MJ, et al. Diagnostic accuracy of fractional flow reserve from anatomic CT angiography. JAMA 2012;308:1237-45.

9 Smith GCS, Pell JP. Parachute use to prevent death and major trauma related to gravitational challenge: systematic review of randomised controlled trials. BMJ 2003;327:1459-61.

10 Yasuda S, Nakao K, Nishimura K, et al. The current status of cardiovascular medicine in Japan- Analysis of a large number of health records from a nationwide claim-based database, JROADDPC. Circ J 2016;80:2327-35.

11 Yasunaga $\mathrm{H}$, Ide $\mathrm{H}$, Imamura $\mathrm{T}$, et al. Impact of the Japanese diagnosis procedure combination-based payment system on cardiovascular medicine-related costs. Int Heart J 2005;46:855-66.

12 Nakamura K. Diagnosis procedure combination database would develop nationwide clinical research in Japan. Circ $J$ 2016;80:2289-90.

13 Yasunaga $\mathrm{H}$, Matsui $\mathrm{H}$, Horiguchi $\mathrm{H}$, et al. [Application of the diagnosis procedure combination (DPC) data to clinical studies]. $J$ Uoeh 2014;36:191-7.

14 lihara K, Nishimura K, Kada A, et al. The impact of comprehensive stroke care capacity on the hospital volume of stroke interventions: a nationwide study in Japan: J-ASPECT study. J Stroke Cerebrovasc Dis 2014;23:1001-18.

15 Chen J, Rathore SS, Wang Y, et al. Physician board certification and the care and outcomes of elderly patients with acute myocardial infarction. J Gen Intern Med 2006;21:238-44.

16 Park S, Sasaki N, Morishima T, et al. The number of cardiologists, case volume, and in-hospital mortality in acute myocardial infarction patients. Int J Cardiol 2013;168:4470-1.
17 Casale PN, Jones JL, Wolf FE, et al. Patients treated by cardiologists have a lower in-hospital mortality for acute myocardial infarction. $J$ Am Coll Cardiol 1998;32:885-9.

18 Spaulding C, Morice M-C, Lancelin B, et al. Is the volumeoutcome relation still an issue in the era of $\mathrm{PCl}$ with systematic stenting? results of the greater Paris area $\mathrm{PCl}$ registry. Eur Heart $J$ 2006;27:1054-60.

19 Curtis JP, Luebbert JJ, Wang Y, et al. Association of physician certification and outcomes among patients receiving an implantable cardioverter-defibrillator. JAMA 2009;301:1661-70.

20 Aversano T, Lemmon CC, Liu L, et al. Outcomes of PCl at hospitals with or without on-site cardiac surgery. N Engl J Med 2012;366:1792-802.

21 Kulkarni VT, Ross JS, Wang Y, et al. Regional density of cardiologists and rates of mortality for acute myocardial infarction and heart failure. Circ Cardiovasc Qual Outcomes 2013;6:352-9.

22 Jong P, Gong Y, Liu PP, et al. Care and outcomes of patients newly hospitalized for heart failure in the community treated by cardiologists compared with other specialists. Circulation 2003;108:184-91.

23 Philbin EF, Jenkins PL. Differences between patients with heart failure treated by cardiologists, internists, family physicians, and other physicians: analysis of a large, statewide database. Am Heart $J$ 2000;139:491-6.

24 Bucholz EM, Butala NM, Normand S-LT, et al. Association of guideline-based admission treatments and life expectancy after myocardial infarction in elderly medicare beneficiaries. J Am Coll Cardiol 2016;67:2378-91.

25 Edep ME, Shah NB, Tateo IM, et al. Differences between primary care physicians and cardiologists in management of congestive heart failure: relation to practice guidelines. J Am Coll Cardiol 1997;30:518-26.

26 Koelling TM, Johnson ML, Cody RJ, et al. Discharge education improves clinical outcomes in patients with chronic heart failure. Circulation 2005;111:179-85.

27 Meltzer D, Manning WG, Morrison J, et al. Effects of physician experience on costs and outcomes on an academic genera medicine service: results of a trial of hospitalists. Ann Intern Med 2002;137:866-74.

28 Bello D, Shah NB, Edep ME, et al. Self-reported differences between cardiologists and heart failure specialists in the management of chronic heart failure. Am Heart J 1999;138:100-7.

29 Bucholz EM, Butala NM, Ma S, et al. Life expectancy after myocardial infarction, according to hospital performance. $N$ Engl J Med 2016;375:1332-42.

30 Cram P, Rosenthal GE, Vaughan-Sarrazin MS. Cardiac revascularization in specialty and general hospitals. N Engl J Med 2005;352:1454-62

31 Badheka AO, Patel NJ, Grover P, et al. Impact of annual operator and institutional volume on percutaneous coronary intervention outcomes: a 5-year United States experience (2005-2009). Circulation 2014;130:1392-406.

32 Ross JS, Normand S-LT, Wang Y, et al. Hospital volume and 30day mortality for three common medical conditions. $N$ Engl J Med 2010;362:1110-8. 